\title{
Some factors involved in complex-picture recognition
}

\author{
MICHAEL J. KIPHART, DOUGLAS D. SJOGREN, and HENRY A. CROSS \\ Colorado State University, Fort Collins, Colorado
}

\begin{abstract}
College students were assigned to factorial combinations of picture context (homogeneous, heterogeneous) and picture color (color, black and white). Subjects were then given homogeneous slides in a picture-recognition test using two distractor types. One distractor type included stimuli not previously shown to the subjects; the other type involved previous stimuli in a different (mirror-image) orientation. Each group was shown 240 pictorial stimuli and was then tested with 48 . Results revealed that picture recognition was not influenced by picture color. However, the results indicated a significant interaction between picture context and distractor type, with performance being significantly influenced only by the homogeneous context with new distractors. Results were discussed in terms of the importance of the initial viewing context.
\end{abstract}

The processes and parameters of human recognition memory have been extensively studied (e.g., Kausler, 1974; Murdock, 1982). Picture recognition involves memory for concrete stimuli such as wilderness scenes, faces, and many other pictorial categories. In a recognition study involving 10,000 heterogeneous photographs, Standing (1973) found high recognition. By extrapolating his results, he conjectured that this memory was for all practical purposes limitless and that if he had employed one million items under the same conditions, his subjects could have recognized approximately 731,400 . Other researchers have found a similar capacity. Shepard (1967) presented 600 picture stimuli, and subjects attained recognition rates as high as $98 \%$ in a two-choice testing procedure. Standing, Conezio, and Haber (1970) provided single presentations of up to 2,568 photographic stimuli and observed approximately $90 \%$ correct recognition.

Not all researchers have encountered this remarkable picture memory capacity. Howe (1967) examined recognition memory for pictorial stimuli presented in homogeneous sets, such as dogs, ships, birds, and trains. The recognition rates for subjects in this study were approximately $72 \%$, which were lower than the rates for heterogeneous stimuli. Goldstein and Chance (1970) utilized a range of complex stimuli and found an inverse relationship between stimulus complexity and recognition performance. The complex homogeneous sets used were pictures of faces, ink blots, and snow crystals. Faces, which were less complex and more familiar, yielded the highest recognition, followed, in descending order, by ink blots and snow crystals, $78 \%, 54 \%$, and $39 \%$, re-

Requests for reprints should be addressed to: Henry A. Cross, Department of Psychology, Colorado State University, Fort Collins, Colorado 80523. spectively. Even though the recognition rates were lower for such complex stimuli, it is apparent that subjects still make reliable recognitions of the stimuli. The lower rates may have been attributable, at least in part, to the homogeneous quality of the stimuli and not simply to their judged complexity.

The purpose of this study was to examine recognition sensitivities for complex homogeneous stimuli that were presented in either a homogeneous or a heterogeneous context. The homogeneous stimuli in both the test set and the homogeneous set were football action photographs that maximized complexity and action. Experiments have shown that learning decreases with an increase of similarity among stimuli (Rundquist, 1968; Underwood \& Ekstrand, 1968) and that stimulus discrimination is an important factor in the formation of associations (Gibson, 1940). The subjects in the present study viewed test pictures of such similarity that one could easily predict difficulty in their making discriminations. It was argued that, under these conditions, overall recognition sensitivity would be seriously impaired.

An additional consideration relates to the context in which the test stimuli were originally viewed. It was hypothesized that, if context were to facilitate the recognition of homogeneous test stimuli, it would do so in the case in which heterogeneous stimuli provided the stimulus backdrop. The reasoning underlying this prediction was that a context of heterogeneous stimuli could afford a greater opportunity for the subject to engage in stimulus differentiation and that superior stimulus differentiation would result in relatively higher recognition than when the same complex and homogeneous stimuli were viewed in the context in which all stimuli were of the same general class.

Also investigated was the role that stimuli that were originally part of the viewing set would have on recognition when they were reversed (shown backward) and 
used as distractors. Standing et al. (1970) showed that changes in picture orientation did not have a significant effect on recognition memory for heterogeneous items, and that subjects tended to recognize the changed stimuli as clearly as they did the originals. This finding indicates that subjects in this study should have had difficulty in rejecting reversed items as distractors, given that these distractors may have been seen as originals and not as distractors. Additionally, the stimulus presentations were either in color or black and white. It was predicted that color would provide an extra discriminating cue and therefore would increase recognition sensitivity. Kruse (1971) showed that subjects learned a complicated surgical procedure better when the procedure was presented in color, rather than in black and white.

\section{METHOD}

\section{Subjects and Design}

The subjects were 63 male and 82 female introductory psychology students at Colorado State University, each of whom received credit for a research requirement. They were tested in a number of groups, with each group corresponding to the particular course section in which they were enrolled. The groups were assigned randomly to the cells of a split-plot design. The between factors of the design were picture context (homogeneous, heterogeneous) and picture color (color, black and white), and the one repeated-measure variable was that of distractor type (old reversed, new).

\section{Stimulus Materials}

Homogeneous viewing set. The homogeneous picture set consisted of 240 football photographs selected from the official files of Colorado State University. The 240 stimuli were assigned randomly to positions in the homogeneous picture set and were video recorded with a 2 -sec stimulus presentation and a $1-\mathrm{sec}$ period of darkness between each stimulus. One homogeneous set was in color, and one was in black and white.

Heterogeneous viewing set. The heterogeneous set contained 240 items: 192 photographs of diverse scenes, people, places, and buildings and 48 football action slides taken from the homogeneous set. The 48 football slides were selected randomly from the homogeneous set and were placed in the same serial positions in the heterogeneous group that they had occupied in the homogeneous set. Two heterogeneous sets were videotaped (under the same time constraints used for the homogeneous set); one set was in color, and the other was in black and white.

Test tape. Of the 48 homogeneous stimuli shared by the picture or viewing sets, 36 were selected randomly for use in the recognition test tape. Twenty-four were used as "old" stimuli, and 12 were employed as "old reversed" distractors. All 48 photographs used for the test tape were football slides; the 48 positions for the test tape were divided into eight blocks of 6 each. Each block contained 3 "old" stimuli and 3 distractors, with the 24 "old" photographs randomly being assigned positions in the test tape, 3 being assigned to each block. For Blocks $2,4,6$, and 8 , the 3 distractors were "old reversed." For Blocks $1,3,5$, and 7 , the 3 distractors were "new" photographs, that is, football action slides not previously shown. Each photograph was video recorded at a 4-sec presentation with a 1-sec interstimulus interval. Test sets were shown in the same color condition as the original viewing sets.

\section{Procedure}

The groups of subjects were tested separately. The experimenter requested that the subjects of each group sit at least one seat apart and in a position in which it would be easy for them

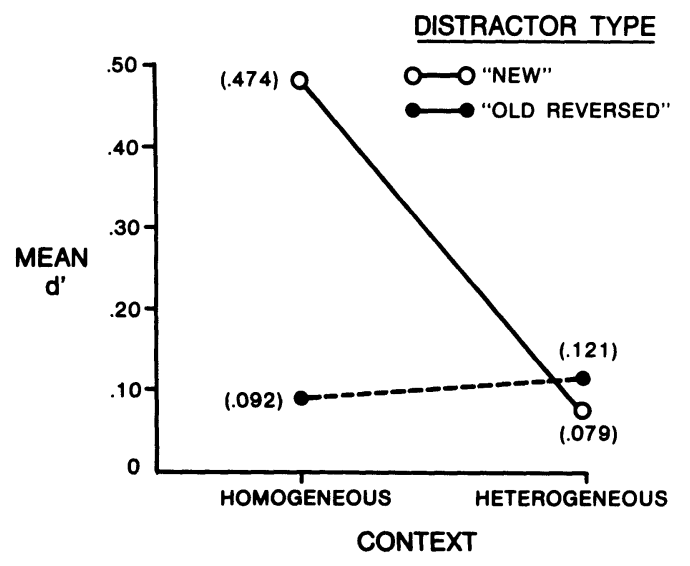

Figure 1. Interaction of context $\mathrm{x}$ distractor types.

to view the monitors located in key positions around the room. The experimenter then instructed the subjects to pay close attention to the pictures that would be shown quickly and consecutively.

After the picture set, the experimenter handed out answer sheets that contained 48 spaces with choices of either "old" or "new." The subjects were told that a test tape would be shown and that each picture of the tape would be either "old" or "new." Pictures that were a part of the previous viewing set were to be regarded as "old," and those that had not appeared in the original set or that were reversed pictures that had appeared in the old set were to be regarded as "new." The dependent measure was $\mathrm{d}^{\prime}$ calculated in accordance with signal detection theory as presented by Swets (1964).

\section{RESULTS}

An analysis of variance was performed on the $\mathrm{d}^{\prime}$, or sensitivity, scores. A significant main effect was found for context $[F(1,141)=6.06, p<.05]$. The means for the homogeneous and heterogeneous contexts were 0.283 and 0.100 , respectively. These means, although different significantly, indicated low sensitivities to the test items. A main effect was also noted for distractor type $[F(1,141)=5.70, p<.05]$. The mean $d^{\prime}$ for the "new" distractors was 0.324 , whereas the mean $d$ ' for the "old reversed" distractors was 0.103. Again, mean sensitivities for these groups were quite low.

A significant interaction was found between context and distractor type $[F(1,141)=7.47, p<.05]$. A Newman-Keuls post hoc test was calculated, and it was found that the mean sensitivity for the homogeneous context with "new" distractors was significantly higher than the other three mean sensitivities, which did not differ among each other. The interaction is illustrated in Figure 1.

\section{DISCUSSION}

The observation that the viewing of complex homogeneous test stimuli may result in low overall recognition scores is consistent with previous results reported by Goldstein and Chance (1970) and further qualifies the more typical finding of an un- 
usual picture-recognition memory. The overall recognition scores of this investigation were, in fact, close to chance level and indicate that, when test stimuli are drawn from unusually homogeneous sets, picture recognition may be quite difficult.

The finding that viewing a set of homogeneous slides in the context of heterogeneous ones did not facilitate subsequent recognition of the homogeneous slides was not expected. In fact, more recognition of the test slides was present when the context was composed of homogeneous slides, although this finding is largely explained by the interaction (context $x$ distractor type) for which the only elevated mean $d^{\prime}$ score was that associated with "new" distractors in the homogeneous context. That subjects had a low d' with "old reversed" distractors in both contexts is predictable from the previous work of Standing et al. (1970). For the subjects who viewed the homogeneous picture sets, the "old reversed" distractors were more difficult than the "new" distractors. However, the subjects who viewed the heterogeneous picture set did not differ significantly in their mean sensitivities to the test stimuli when distractor type was examined.

A possible explanation for the elevated mean $d$ ' scores among subjects who were exposed to "new" distractors after having viewed stimuli in the homogeneous context is attentional in nature. When the subject views only complex homogeneous stimuli, it quickly becomes apparent that test stimuli must necessarily be drawn from this population and that all stimuli are carefully observed. With an increasing number of stimuli, the necessity to make ever more refined observations is apparent. By contrast, subjects viewing the heterogeneous set see less need to make subtle differentiations among the test slides, since only 48 of the 240 total stimuli are homogeneous. Alternatively, in the heterogeneous context, it is likely that the similarities among the homogeneous slides are accentuated and postexperiment discussions with subjects confirmed this.

Barnard, Breeding, and Cross (1984) have recently observed that the recognition of objects (shown directly rather than pictorially) and the decline of recognition for these same objects over a 1-week retention interval are partially dependent upon specific object characteristics as revealed by cluster analysis of rated stimulus dimensions. The present results provide still another factor, the homogeneous or heterogeneous nature of the context in which complex homogeneous pictures are originally viewed, which influences subsequent recognition performance. Future research should examine not only recognition for homogeneous items embedded in homogeneous and heterogeneous contexts, but should explore the recognition of heterogeneous items as well. Beyond this, the influence upon subsequent recognition of the relative homogeneity or heterogeneity of the total viewing set should be explored.

\section{REFERENCES}

Barnard, W. A., Breeding, M., \& Cross, H. A. (1984). Object recognition as a function of stimulus characteristics. Bulletin of the Psychonomic Society, 22, 15-18.

Gibson, E. J. (1940). A systematic application of the concepts of generalization and differentiation to verbal learning. Psychological Review, 47, 196-229.

Goldstein, A. G., \& Chance, J. E. (1970). Visual recognition memory for complex configurations. Perception \& Psychophysics, 9, 237-241.

Howe, M. J. A. (1967). Recognition memory for photographs in homogeneous sequences. Perceptual and Motor Skills, 24, 1181-1182.

KAUSLE R, D. H. (1974). Psychology of verbal learning and memory. New York: Academic Press.

KRUSE, W. D. (1971). Comparison of a color film and a black and white videotape on learning a surgical procedure. Unpublished master's thesis, Colorado State University, Fort Collins.

Murdock, B. B. (1982). Recognition memory. In C. R. Puff (Ed.), Handbook of research methods in human memory and cognition. New York: Academic Press.

RUNDQUYST, W. N. (1968). Functions relating intralist similarity in paired-associate learning. Journal of Experimental Psychology, 7, 549-553.

SHEPARD, R. (1967). Recognition memory for words, sentences, and pictures. Journal of Verbal Learning and Verbal Behavior, 6, 156-163.

StANDING, L. (1973). Learning 10,000 pictures. Quarterly Journal of Experimental Psychology, 25, 207-222.

Standing, L., Conezio, H., \& Haber, R. N. (1970). Perception and memory for pictures: Single-trial learning of 2,500 visual stimuli. Psychonomic Science, 19, 73-74.

SwETS, J. A. (1964). Signal detection theory and recognition in human observers. New York: Wiley.

UNDERWOod, B. J., \& Ekstrand, B. R. (1965). Differentiation among stimuli as a factor in transfer performance. Journal of Verbal Learning and Verbal Behavior, 4, 447-462.

(Manuscript received for publication December 14, 1983.) 